VOL. 41 (1990) [123-134]

\title{
A HILBERT ALGEBRA OF HILBERT-SCHMIDT QUADRATIC OPERATORS
}

\section{J.C. Amson and N. Gopal Reddy}

A quadratic operator $Q$ of Hilbert-Schmidt class on a real separable Hilbert space $H$ is shown to be uniquely representable as a sequence $\left(L^{k}\right)^{k \in I}$ of self-adjoint linear operators of Hilbert-Schmidt class on $H$, such that $Q(x)=\sum_{k}\left\langle L^{k} x, x\right\rangle u_{k}$ with respect to a Hilbert basis $\left(u_{k}\right)_{k \in I},(I \subseteq N)$. It is shown that with the norm $|\|Q\||=\left(\sum_{k}\left\|L^{k}\right\|^{2}\right)^{(1 / 2)}$ and inner-product $\langle\langle\langle Q, P\rangle\rangle\rangle=\sum_{k}\left\langle\left\langle L^{k}, M^{k}\right\rangle\right\rangle$, together with a multiplication defined componentwise through the composition of the linear components, the vector space of all Hilbert-Schmidt quadratic operators $Q$ on $H$ becomes a linear $\mathrm{H}^{*}$-algebra containing an ideal of nuclear (trace class) quadratic operators. In the finite dimensional case, each $Q$ is also shown to have another representation as a block-diagonal matrix of Hilbert-Schmidt class which simplifies the practical computation and manipulation of quadratic operators.

\section{INTRODUCTION}

The purpose of this paper is to show how a space of quadratic operators (that is homogeneous polynomial operators of degree 2 , (see for example $[3,4,7,8]$ ) can be given a multiplication under which it becomes a linear algebra. The construction is new in that it does not make use of the composition of quadratic operators, nor of the composition of the linear maps associated with the tensor product of the quadratic operator's domain. The former attempt fails because the composition of two quadratic operators is generally of degree 4. The latter fails because even though a quadratic operator from one vector space $E$ to another $F$ is associated with a unique symmetric bilinear operator on the cartesian product $E \times E \rightarrow F$ and hence with a unique linear operator on the tensor product $E \otimes E \rightarrow F$, those linear operators may only be composed in the extremely unusual case where $F=E \otimes E$. More elaborate nonlinear algebras of homogeneous polynomial operators do exist of course [2], but these form a very different topic from the one studied here.

\section{Received 22 February 1989}

The second author wishes to acknowledge the assistance of Osmania University in making available sabbatical leave and the assistance of the University of St Andrews and the Department of Mathematical Sciences in providing study facilities during his visit. Both authors wish to thank the Rev. D.D.Galbraith, University Chaplain, for practical assistance, and an anonymous fund for additional financial support.

Copyright Clearance Centre, Inc. Serial-fee code: 0004-9729/90 \$A2.00+0.00. 
To be able to include bounded (continuous) quadratic operators we first develop our ideas in the context of Hilbert space theory, and further specialise our treatment to the case where the quadratic operators are the 2 nd degree analogues of linear HilbertSchmidt operators $[5,6,9,10]$.

We represent a Hilbert-Schmidt quadratic operator as a square-summable sequence $\left(L^{k}\right)^{k \in I}$ of Hilbert-Schmidt linear operators on $H$. The linear components act as coordinate quadratic forms through the (real) bilinear inner-product. We form a Hilbert space of all such operators on $H$ in a natural way as a generalised sequence space $l^{2}(I, H S L(H))$. We then construct from this an $\left(\mathrm{H}^{*}\right)$ Hilbert algebra $[1,5]$ by introducing a componentwise multiplication which makes it a function algebra. The product of two Hilbert-Schmidt quadratic operators is then a nuclear (trace class) quadratic operator $[6,8]$, since each of its components is automatically a nuclear linear operator. Finally, we show how the members of this algebra may also be represented in the finite dimensional case by Hilbert-Schmidt real matrices of a special 'block diagonal' type.

The applications of these general ideas to cases involving real Hilbert spaces of low dimension will obviously have many connections with, for example, extensions of the known theory of the geometry of bilinear and quadratic forms. There will also be applications in the field of numerical and symbolic computations involving convenient representations of the finite rank quadratic operators arising in 2 nd-degree integral and algebraic equations.

\section{Notation AND PRELIMINARIES}

Let $H$ be a real, separable Hilbert space with Hilbert basis $\left\{u_{i}\right\}_{i \in I}$, where $I \subseteq \mathbf{N}$. $|I|$ is the cardinality of $I$. We work in a real space because we require a bilinear innerproduct; the case of a complex space with sesquilinear inner-product needs additional study. The separability of $H$ is only for convenience; our results hold for an arbitrary real Hilbert space. Let $L(H)$ be the space of all bounded linear operators on $H$. We denote by $H S L(H)$ the Banach space of all Hilbert-Schmidt linear operators on $H$; the uniform norm and Hilbert-Schmidt norm will be denoted by $\|$.$\| and \|. \mid\|$, respectively.

We recall that a triply-indexed real array $\mathrm{a}=\left[a_{i j}^{k}\right]_{i, j \in I}^{k \in I}$ is called a 2-matrix of size $|I| \times|I| \times|I|$. For each $k \in I$, the sub-array $\left[a_{i j}^{k}\right]_{i, j \in I}$ is an ordinary matrix (1-matrix). We introduce the notation:

$$
\left.\|\mathbf{a}\|\right|^{2}=\sum_{k} \sum_{i} \sum_{j}\left|a_{i j}^{k}\right|^{2} .
$$

By analogy with the ordinary Hilbert-Schmidt matrix, we make the following definition:

Definition 1: A 2-matrix a is said to be a Hilbert-Schmidt 2-matrix if $\|\mathbf{a}\| \|^{2}<$ $+\infty$. The set of all Hilbert-Schmidt 2-matrices of size $|I| \times|I| \times|I|$ is denoted by $H S 2 M(I)$ or $H S 2 M$ if $I$ is understood. 
HS2M is evidently a Banach space under the norm $\|\mid \mathbf{a}\|$, and a Hilbert space under the inner-product

$$
\langle\langle\mathbf{a}, \mathbf{b}\rangle\rangle=\sum_{k}\left\langle\left\langle\mathbf{a}^{k}, \mathbf{b}^{k}\right\rangle\right\rangle=\sum_{k} \sum_{i} \sum_{j} a_{i j}^{k} b_{i j}^{k} .
$$

For each $\mathbf{a} \in H S 2 M$, and for each $k \in I$, the sub-matrix $\mathbf{a}^{k}$ is plainly an ordinary Hilbert-Schmidt matrix, with

$$
\left|\left\|\mathbf{a}^{k}\right\|\right|^{2}=\sum_{i} \sum_{j}\left|a_{i j}^{k}\right|^{2}
$$

and we can denote each $\mathbf{a} \in H S 2 M$ as a sequence $\mathbf{a}=\left(\mathbf{a}^{k}\right)^{k \in I}$.

We recall too that the space of all real Hilbert-Schmidt matrices HS1M, $|\|\cdot\||$, is isometric-isomorphic to the Hilbert space $H S L(H)$ of Hilbert-Schmidt linear operators on $H$ under the identification $\left[a_{i j}\right] \leftrightarrow A$ given by:

$$
\forall i, j \in I, \quad a_{i j}=\left\langle A u_{i}, u_{j}\right\rangle
$$

Hence

$$
(\forall k \in I), \quad\left\|\left|\mathbf{a}^{k}\right|\right\|^{2}=\left\|\left|A^{k}\right|\right\|^{2}=\sum_{i} \sum_{j}\left|\left\langle A^{k} u_{i}, u_{j}\right\rangle\right|^{2}=\sum_{i}\left\|A^{k} u_{i}\right\|^{2},
$$

where each $A^{k}$ is the linear Hilbert-Schmidt operator corresponding to the HilbertSchmidt matrix $\mathbf{a}^{k}$.

This identification clearly extends to an isometric-isomorphism between the space HS2M of Hilbert-Schmidt 2-matrices $\mathbf{a}=\left(\mathbf{a}^{k}\right)^{k \in I}$ and the Hilbert sum $\bigoplus_{k \in I} H S L(H)$ of all sequences $A=\left(A^{k}\right)^{k \in I}$ given the norm $\|A\| \mid$ via:

$$
\|A\|\left\|^{2}=\sum_{k}\right\| A^{k}\|\|^{2}=\sum_{k} \sum_{i}\left\|A^{k} u_{i}\right\|^{2}=\sum_{k} \sum_{i} \sum_{j}\left|\left\langle A^{k} u_{i}, u_{j}\right\rangle\right|^{2},
$$

(the order of summations being interchangeable because all the series are real and positive and hence absolutely convergent), and inner-product:

$$
\langle\langle\langle A, B\rangle\rangle\rangle=\sum_{k}\left\langle\left\langle A^{k}, B^{k}\right\rangle\right\rangle=\sum_{k} \sum_{i} \sum_{j}\left\langle A^{k} u_{i}, B^{k} u_{j}\right\rangle,
$$

(using an obvious notation). We shall show below that each such square-summable sequence $A$ of linear Hilbert-Schmidt operators $A^{k}$ determines a bounded quadratic operator - indeed a Hilbert-Schmidt quadratic operator - on $H$. We shall also prefer to replace the Hilbert sum $\bigoplus_{k \in I} H S L(H)$ by its equivalent generalised sequence space $l^{2}(I, H S L(H))$ of square-norm-summable sequences of operators $A^{k} \in H S L(H)$. 


\section{DEFINITION AND REPRESENTATION OF A HILBERT-SCHMIDT QUADRATIC OPERATOR}

Let $I \subseteq \mathrm{N}$; let $\left\{u_{i}\right\}_{i \in I}$ be a Hilbert basis for $H$. Let $Q: H \rightarrow H$ be a bounded (and hence continuous) quadratic operator on $H$ with its associated (unique) continuous symmetric bilinear operator $\widehat{Q}: H \times H \rightarrow H$ such that $Q(x)=\widehat{Q}(x, x)$ identically, and $\|Q\|=\|\widehat{Q}\|$. Let $x=\sum_{i} x^{i} u_{i}$, and $z=\sum_{k} z^{k} u_{k}$ be vectors in $H$, such that $Q(x)=z$ where the coordinates $z^{k}$ of $z$ are uniquely determined by $Q$. We note of course that this 'uniqueness' is only with respect to the Hilbert basis chosen for $H$, or equivalently 'to within a unitary transformation' of $H$. Now

$$
\begin{aligned}
\sum_{j} z^{j} u_{j}=z & =Q(x)=\hat{Q}(x, x)=\widehat{Q}\left(\sum_{i} x^{i} u_{i}, \sum_{j} x^{j} u_{j}\right) \\
& =\sum_{i} \sum_{j}\left(\hat{Q}\left(u_{i}, u_{j}\right) x^{i} x^{j}\right) \\
& =\sum_{i} \sum_{j}\left(\sum_{k} q_{i j}^{k} u_{k}\right) x^{i} x^{j} \text { (say) } \\
& =\sum_{k}\left(\sum_{i} \sum_{j} q_{i j}^{k} x^{i} x^{j}\right) u_{k} .
\end{aligned}
$$

Since coordinates are unique, and, for each $k \in I$, the scalars $q_{i j}^{k}$ form a matrix $\mathbf{q}^{k}$ uniquely determined by $Q$, it follows that each $z^{k}=\sum_{i} \sum_{j} q_{i j}^{k} x^{i} x^{j}$, and is therefore the value of a unique bounded quadratic functional or form $Q^{k}$. Using familiar matrixvector notation we have:

$$
z^{k}=\left(x_{1}, x_{2}, \ldots\right)\left[q_{i j}^{k}\right]\left(\begin{array}{c}
x_{1} \\
x_{2} \\
\vdots
\end{array}\right)=x^{t r} q^{k} x
$$

From the uniqueness of the Fourier representation

$$
z=Q(x)=\sum_{k}\left\langle Q(x), u_{k}\right\rangle u_{k}=\sum_{k} z^{k} u_{k}
$$

we also have the alternative representation (in virtue of the Riesz Theorem):

$$
z^{k}=\left\langle L^{k} x, x\right\rangle
$$

where $L^{k}$ is the bounded linear operator corresponding to the bounded quadratic functional $Q^{k}$ given by $Q^{k}=\left\langle Q(x), u_{k}\right\rangle$. Of course, $L^{k}$ can be assumed to be self-adjoint, in which case it is unique. We also note that these results hold precisely because the inner-product in our real Hilbert space $H$ is bilinear and not sesquilinear. 
Proposition 1. For each $k \in I$, with $L^{k}$ and $Q^{k}$ as defined above, we have

$$
\left\|Q^{k}\right\|=\left\|L^{k}\right\| \leqslant \quad\left\|L^{k}\right\||=|\left\|Q^{k}\right\| \mid
$$

Proof: $\left|Q^{k}(x)\right|=\left|\left\langle L^{k} x, x\right\rangle\right| \leqslant\left\|L^{k}\right\|,(\forall\|x\|=1)$, hence $\left\|Q^{k}\right\| \leqslant\left\|L^{k}\right\|$; let $\hat{Q}^{k}$ be the unique symmetric bilinear functional associated with $Q$; then

$$
\left\|Q^{k}\right\|=\left\|\widehat{Q}^{k}\right\| \geqslant\left|\widehat{Q}^{k}(x, y)\right| /\|x\|\|y\|=\left|\left\langle L^{k} x, y\right\rangle\right| /\|x\|\|y\|,
$$

in particular for $y=L^{k}(x) \neq 0$ we have

$$
\left\|Q^{k}\right\| \geqslant\left\|L^{k} x\right\|^{2} /\|x\|\left\|L^{k}(x)\right\|=\left\|L^{k} x\right\| /\|x\|,
$$

taking sup over all $x \neq 0$ gives $\left\|Q^{k}\right\| \geqslant\left\|L^{k}\right\|$, whence equality. The second equality follows from the fact that both $Q^{k}$ and $L^{k}$ are represented by the same Hilbert-Schmidt 1-matrix $\mathbf{q}^{k}=\left[\boldsymbol{q}_{i j}^{k}\right]$. The majorising of the uniform norm by the Hilbert-Schmidt norm is a standard result.

We observe that the bounded quadratic operator $Q: H \rightarrow H$ has two unique (related) representations, one as a sequence $L=\left(L^{k}\right)^{k \in I}$ of bounded self-adjoint linear operators, the other as a sequence $\mathbf{q}=\left(\mathbf{q}^{k}\right)^{k \in I}$ of symmetric matrices $\mathbf{q}^{k}=\left[q_{i j}^{k}\right]_{i, j \in I}$ which itself forms a 2-matrix $\left[q_{i j}^{k}\right]_{i, j \in I}^{k \in I}$. Using Parseval's equality we have:

$$
\|Q(x)\|^{2}=\sum_{k}\left|z^{k}\right|^{2}=\sum_{k}\left|\left\langle L^{k} x, x\right\rangle\right|^{2} \leqslant \sum_{k}\left\|L^{k}\right\|^{2}\|x\|^{2},
$$

from which we get $\|Q\|^{2} \leqslant \sum_{k}\left\|L^{k}\right\|^{2} \leqslant \sum_{k}\left|\left\|L^{k}\right\|\right|^{2}$. But there is no reason why, in general, either sum on the right should be finite. The class of all those quadratic operators for which at least one sum were finite would plainly be an interesting one. In particular, for the remainder of this study, we shall insist on both sums being finite, and for reasons which will be immediately obvious we make this definition:

DEFINITION 2: A bounded quadratic operator $Q: H \rightarrow H$ will be said to be a Hilbert-Schmidt quadratic operator if the series $\sum_{k}\left|\left\|L^{k}\right\|\right|^{2}$ is convergent; in which case its sum is denoted by $\|Q\| \|^{2}$. The set of all such Hilbert-Schmidt quadratic operators on $H$ will be denoted by $H S Q(H)$.

We note that each Hilbert-Schmidt quadratic operator $Q \in H S Q(H)$ has representations (i) $\left(L^{k}\right)^{k \in I}$ as a unique square-norm-summable sequence of self-adjoint HilbertSchmidt linear operators on $H$, and (ii) $\left(\mathbf{q}^{k}\right)^{k \in I}$ as a unique square-norm-summable sequence of symmetric Hilbert-Schmidt matrices. We shall refer to these as the sequence 
representations of $Q$, and write $Q \sim\left(L^{k}\right)^{k \in I} \sim\left(\mathbf{q}^{k}\right)^{k \in I}$. Thus, the adjoint of $Q$, $Q^{*} \stackrel{\text { def }}{\sim}\left(L^{k *}\right)^{k \in I}=\left(L^{k}\right)^{k \in I} \sim Q$. Hence every $Q \in H S Q(H)$ is self-adjoint.

The proofs of the results in the next Theorem are then readily obtained from the above, the discussion of Hilbert-Schmidt 2-matrices in Section 1, and the standard properties of linear Hilbert-Schmidt operators.

THEOREM 1. Let $H$ be a real Hilbert space, with Hilbert basis $\left\{u_{i}\right\}_{i \in I}$. If $Q \sim$ $\left(L^{k}\right)^{k \in I} \sim\left(\mathbf{q}^{k}\right)^{k \in I}$ is a Hilbert-Schmidt quadratic operator in HSQ $(H)$, then

$$
\begin{aligned}
\|Q\|^{2} & \leqslant \sum_{k}\left\|L^{k}\right\|^{2} \leqslant \sum_{k}\left|\left\|L^{k}\right\|\right|^{2}=\left.\|Q\|\right|^{2}=\left.\left\|Q^{*}\right\|\right|^{2} . \\
\left.\|Q\|\right|^{2} & =\left.\sum_{k}\left\|\mathbf{q}^{k}\right\|\right|^{2}=\sum_{k} \sum_{i} \sum_{j}\left|q_{i j}^{k}\right|^{2} . \\
\left.\|Q\|\right|^{2} & =\sum_{k}\left\|L^{k} u_{k}\right\|^{2}=\sum_{k} \sum_{i} \sum_{j}\left|\left\langle L^{k} u_{i}, u_{j}\right\rangle\right|^{2} .
\end{aligned}
$$

If $P \sim\left(M^{k}\right)^{k \in I} \sim\left(\mathrm{p}^{k}\right)^{k \in I}$ and $R \sim\left(N^{k}\right)^{k \in I} \sim\left(\mathbf{r}^{k}\right)^{k \in I}$ are also Hilbert-Schmidt quadratic operators in $H S Q(H)$, then

$$
\begin{aligned}
\langle\langle(Q, P\rangle)\rangle & \stackrel{\text { def }}{=} \sum_{k}\left\langle\left\langle Q^{k}, P^{k}\right\rangle\right\rangle=\sum_{k} \sum_{i}\left\langle L^{k} u_{i}, M^{k} u_{i}\right\rangle . \\
\langle(\langle Q, Q\rangle\rangle\rangle & =\|\| Q \|\left.\right|^{2} . \\
\langle\langle\langle Q P, R\rangle\rangle\rangle & =\left\langle\left\langle\left\langle P, Q^{*} R\right\rangle\right\rangle\right\rangle=\left\langle\left\langle\left\langle Q, R P^{*}\right\rangle\right\rangle\right\rangle .
\end{aligned}
$$

THEOREM 2. The set HSQ(H) of all Hilbert-Schmidt quadratic operators on a Hilbert space $H$ with a Hilbert basis $\left\{u_{i}\right\}_{i \in I}$ is a vector space under componentwise operations, on which $Q \mapsto\|Q\|$ is a norm; furthermore, $H S Q(H)$, $\|\cdot\| \|$ is a Banach space. It is also a real Hilbert space under the inner-product $(Q, P) \mapsto\langle(\langle Q, P\rangle\rangle\rangle$, isometric-isomorphic to the space of real Hilbert-Schmidt 2-matrices $\left[q_{i j}^{k}\right]_{i, j \in I}^{k \in I}$ where the cardinality of the index set $I$ and the dimension of $H$ are the same.

Proof: That $H S Q(H)$ is a vector space, and $\|\cdot\| \mid$ is a norm, follows at once by the usual computations using Minkowski's inequality. That $\langle(\langle, \mid\rangle\rangle$ is an inner-product also follows by the usual computations using Hölder's inequality. The completeness of the norm follows at once by appealing to the identification of $H S Q(H)$ with $H S 2 M$ the space of all Hilbert-Schmidt 2-matrices which itself is isometric-isomorphic to the real sequence space $l^{2}(I, \mathbf{R})$. Alternatively, the standard strategy for proving that the classical Hilbert space $l^{2}(I, \mathbf{R})$ is complete can be directly applied, detail for detail, to 
$H S Q(H)$ the only changes needed being that the modulus $|$.$| and the vector norm \|$. are everywhere replaced by the uniform norm $\|\cdot\|$ and the Hilbert-Schmidt norm ||.|\|, respectively.

REMARK. The analogy between the space $H S Q(H)$ regarded as a space of sequences $\left(L^{k}\right)$ and the classical sequence space $l^{2}(I, \mathbf{R})$ referred to in the previous proof will now be exploited further in the next section when we come to impose an additional operation of 'componentwise multiplication' on $H S Q(H)$ which gives it the structure of a normed algebra, a linear $\mathrm{H}^{*}$-algebra, in fact.

\section{An H*-Algebra of Hilbert-Schmidt Quadratic Operators}

Let $H S Q(H)$ be the Hilbert space of Hilbert-Schmidt quadratic operators on a real Hilbert space $H$ as constructed in the previous section. It is now clear that one representation of $H S Q(H)$ is as a generalised sequence space $l^{2}(I, H S L(H))$ of squarenorm-summable sequences $Q \sim\left(L^{k}\right)^{k \in I}$ of self-adjoint linear Hilbert-Schmidt operators on $H$, the vector space operations being defined componentwise as usual. We now introduce another internal law of composition on this space by defining the product of two Hilbert-Schmidt quadratic operators componentwise.

DEFINITION 3: By multiplication in HSQ $(H)$ we mean the operation, denoted by - and defined as follows:

$$
\forall Q \sim\left(L^{k}\right)^{k \in I}, \forall P \sim\left(M^{k}\right)^{k \in I}, \quad Q \bullet P \stackrel{\text { def }}{=}\left(L^{k} \circ M^{k}\right)^{k \in I} .
$$

Thus the product of two Hilbert-Schmidt quadratic operators is constructed componentwise from the composition of their self-adjoint linear Hilbert-Schmidt components. Of course the composition of two self-adjoint linear operators need not be self-adjoint, since, for example the product of two $2 \times 2$ real symmetric matrices need not be symmetric. If we insisted on self-adjoint components for a Hilbert-Schmidt quadratic operator then we would have to symmetrise each composed component by taking half the sum of itself and its Hilbert-adjoint. But it is not hard to verify that this symmetrisation will destroy the $\mathrm{H}^{*}$-algebra conditions (the so-called 'Ambrose' conditions) listed as point (6) in Theorem 1.

However, the self-adjointness of the components is not an essential condition, anymore than the condition that a bilinear functional must be symmetric in order to produce a quadratic functional by restricting it 'to the diagonal': $q(x)=b(x, x)$. In that situation it is only the symmetric-part $b$, of the bilinear $b$ that is involved in producing the quadratic, the anti-symmetric part $b_{a}$ being identically zero on the diagonal. In the same way, it is only the self-adjoint part $L$, of a linear component $L$ that is effective in 
being identified with the quadratic operator, the anti-self-adjoint part $L_{a}$ contributing nothing to the quadratic operator's functional properties. In that sense, our representations of Hilbert-Schmidt quadratic operators as sequences of linear Hilbert-Schmidt operators are strictly speaking unique 'only to within self-adjointness'.

Proposition 2. The norm ||$\cdot|| \mid$ in the space HSQ(H) of Hilbert-Schmidt quadratic operators on $H$ is sub-multiplicative:

$$
\|\| Q \bullet P\||\leqslant\|Q\|| \cdot\| P \| \mid
$$

hence $H S Q(H)$ is closed under the multiplication (•), and multiplication is continuous on $H S Q(H)$.

Proof: Let $Q \sim\left(L^{k}\right)^{k \in I}, P \sim\left(M^{k}\right)^{k \in I} \in H S Q(H)$. Assume $I=\mathbf{N}$, then for each $N \in \mathbf{N}$,

$$
\sum_{k=1}^{N}\left\|L^{k} \circ M^{k}\right\|^{2} \leqslant \sum_{k=1}^{N}\left\|L^{k}\right\|^{2} \cdot\left\|M^{k}\right\|^{2}
$$

(since $L^{k}, M^{k} \in$ the same $H S L(H)$, an $H^{*}$-algebra). Hence the partial sums on the lefthand-side are bounded above by the product $\sum_{k}\left|\left\|L^{k}\right\|\right|^{2} \cdot \sum_{k}\left|\left\|M^{k}\right\|\right|^{2}=\|\| Q\||\cdot\|P\||$ it follows that the series $\sum_{k}\left\|L^{k} \circ M^{k}\right\|^{2}$ is convergent, with sum $\leqslant\|\| Q\|\mid \cdot\| P\|\|$, so that we have $\|Q \bullet P\|\|\leqslant\| Q\|\| \cdot\|P\|$, as asserted. The other conclusions follow immediately.

THEOREM 3. The Hilbert space $H S Q(H)$, equipped with the multiplication (•) is an $\mathrm{H}^{*}$-algebra.

Proof: All that remains to be verified is (i) that the multiplication $\bullet$ distributes componentwise over addition + and commutes with multiplication by scalars, so that $(H S Q(H), \bullet)$ becomes an algebra, and (ii) that the 'Ambrose' conditions (Theorem $1(6)$ ) are satisfied. Both of these verifications are simply routine calculations since all the required properties are inherited componentwise from the analogous properties in each component algebra $H S L(H)$ of Hilbert-Schmidt linear operators on $H$.

REMARK. The algebra $H S Q(H)$ constructed above is, of course, a particular example of a Function Algebra. It can have a multiplicative unit if and only if the dimension of the space $H$ is finite (that is if the index set $I$ of the sequence space $H S Q(H)$ is finite). If there is a unit Hilbert-Schmidt quadratic operator $I$ say, each of its component linear operators $I^{k}$ would necessarily be a unit in the algebra $H S L(H)$ of Hilbert-Schmidt linear operators; but that can happen if and only if the space $H$ is finite dimensional. In this case each component algebra $H S L(H)$ coincides with the entire algebra of bounded linear operators on $H$, and the $\mathrm{H}^{*}$-algebra $H S Q(H)$ coincides with the entire space of bounded quadratic operators on $H$. 
We now introduce the idea of a nuclear (or trace class) quadratic operator. We begin with the fact $[6,9]$ that a bounded linear operator $N$ on $H$ is a nuclear if and only if it is the product $L \circ M$ of two Hilbert-Schmidt linear operators $L, M$.

Proposition 3. Each product $R=Q \bullet P$ of two Hilbert-Schmidt quadratic operators $Q, P \in H S Q(H)$ has nuclear components.

Proof: Let $Q \sim\left(L^{k}\right)^{k \in I}, P \sim\left(M^{k}\right)^{k \in I}, R \sim\left(N^{k}\right)^{k \in I}$. Then each $N^{k}=$ $L^{k} \circ M^{k}$ is the composition of two Hilbert-Schmidt linear operators and hence a nuclear linear operator on $H$.

Since the algebra $H S Q(H)$ is closed under multiplication $(\bullet)$, the next definition is meaningful:

DEFINITION 4: A Hilbert-Schmidt quadratic operator $N \sim\left(N^{k}\right)^{k \in I} \in H S Q(H)$ is said to be a nuclear quadratic operator if and only if each of its linear components $N^{k}$ is nuclear. We denote by $N Q(H)$ the subset of $H S Q(H)$ consisting of all nuclear quadratic operators on $H$.

The next theorem follows directly from the componentwise structure of the function algebra $H S Q(H)$ and the fact that the nuclear linear operators on $H$ form an ideal in the algebra $H S L(H)$ of Hilbert-Schmidt linear operators on $H$.

THEOREM 4. The set $N Q(H)$ of nuclear quadratic operators on $H$ is an ideal in the Hilbert algebra $H S Q(H)$ of Hilbert-Schmidt quadratic operators on $H$. The ideal is proper if and only if $H$ has infinite dimension.

It is also possible to extend the notion of multiplication $(\bullet)$ in $H S Q(H)$ to include a situation denoted by $\bar{A} \bullet Q \bullet \bar{B}$ where $\bar{A}, \bar{B}$ denote constant sequences $\left(A^{k}\right)^{k \in I}, A^{k}=$ $A,\left(B^{k}\right)^{k \in I}, B^{k}=B$ where $A, B \in L(H)$ are arbitrary bounded linear operators on $H$. If $Q \sim\left(L^{k}\right)^{k \in I}$, we simply set

$$
\bar{A} \bullet Q \cdot \bar{B}=\left(A \circ L^{k} \circ B\right)^{k \in I}
$$

Since $\left\|A \circ L^{k} \circ B\right\| \leqslant\|A\| \cdot\left\|L^{k}\right\| \cdot\|B\| \leqslant\|A\| \cdot\left\|L^{k}\right\|\|\cdot\| B \|, \quad$ we readily compute that

$$
\|\bar{A} \bullet Q \cdot \bar{B}\| \mid \leqslant\|A\| .\|Q\|\|\cdot\| B \|,
$$

from which it follows that

$$
\bar{A} \bullet Q \bullet \bar{B} \in H S Q(H) .
$$

In particular, these results hold when $A=U^{-1}, B=U$ and $U$ is any unitary (changeof-basis) operator on $H$.

The obvious implications of this extended multiplication together with the concept of quadratic operator ideals - exploiting componentwise the properties of norm ideals of von Neumann-Schatten classes [9] - will be studied in a subsequent paper on function algebras of compact quadratic operators. 


\section{Block Diagonal Matrix Representation}

In virtue of Ambrose's theorem ([1]; [5, p.1013]), our $H^{*}$-algebra (HSQ(H), •) is topologically and algebraically isomorphic to an algebra of Hilbert-Schmidt operators on some Hilbert space. We now show that for $(H S Q(H), \bullet)$ in the special case where $H$ has finite dimension, one such algebra is a linear matrix algebra on the Hilbert sum $H S L\left(\bigoplus_{k \in I} H\right)$ of $|I|$ copies of the Hilbert space $H$. The matrices in question are Hilbert-Schmidt matrices of a special 'block-diagonal' kind. The construction can be extended (in principle rather than in practice) to the general case where $H$ has infinite dimension.

Let $H$ have finite dimension $N$ and, without loss of generality, assume that $I=$ $\{1,2, \ldots, N\}$. Let $Q \in H S Q(H)$ have its representation $\left(\mathbf{q}^{1}, \mathbf{q}^{2}, \ldots, \mathbf{q}^{N}\right)$ as a finite sequence of Hilbert-Schmidt matrices $q^{k}$ with respect to a Hilbert basis $\left\{u_{i}\right\}_{i \in I}$ for $H$. The block-diagonal representation of $Q$ is easiest to visualise when $N$ is small: suppose $H=\mathbf{R}^{2}$, with a Hilbert basis $\left\{u_{1}=(1,0), u_{2}=(0,1)\right\}$ so that $Q=\left(\mathbf{q}^{1}, \mathbf{q}^{2}\right)$, with 2-matrix representation

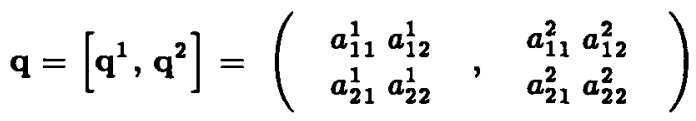

We re-write these two component matrices $q^{1}, q^{2}$ as block-diagonal entries in a $4 \times 4$ ordinary matrix, and fill the remaining off-diagonal blocks with 0 's :

$$
\left(\begin{array}{cccc}
a_{11}^{1} & a_{12}^{1} & 0 & 0 \\
a_{21}^{1} & a_{22}^{1} & 0 & 0 \\
0 & 0 & a_{11}^{2} & a_{12}^{2} \\
0 & 0 & a_{21}^{2} & a_{22}^{2}
\end{array}\right)
$$

In general the block-diagonal representation of $Q$, denoted by $B D[Q]$, will be

$$
B D[Q]=\left(\begin{array}{cccc}
{\left[\mathbf{q}^{1}\right]} & {[0]} & \ldots & {[\mathbf{0}]} \\
{[0]} & {\left[\mathbf{q}^{2}\right]} & \ldots & {[\mathbf{0}]} \\
\vdots & \vdots & \ddots & {[\mathbf{0}]} \\
{[0]} & {[\mathbf{0}]} & \ldots & {\left[\mathbf{q}^{N}\right]}
\end{array}\right)
$$

Such a block-diagonal matrix (in which, in general, the Hilbert-Schmidt diagonal blocks $\left[\mathrm{q}^{k}\right]$ need not be symmetric) will be called an $H S$ block-diagonal matrix or $H S B D$ matrix for short; its size is $|I|^{2} \times|I|^{2}=N^{2} \times N^{2}$. That the matrix-product of two equally sized $H S B D$ matrices is another $H S B D$ of same size follows from the facts that the block-diagonal structure is preserved by matrix multiplication and the product of two Hilbert-Schmidt matrices is another Hilbert-Schmidt matrix. Of course the 
subspace of those $H S B D$ matrices with all their diagonal blocks $\mathbf{q}^{k}$ symmetric is not closed under matrix multiplication, since as noted earlier the product of two symmetric matrices need not be symmetric.

Proposition 4. Let $\|B D[Q]\| \mid$ be the Hilbert-Schmidt norm of an $B S B D$ matrix $B D[Q]$ representing a quadratic operator $Q \in H S Q(H)$. Then

$$
|\|B D[Q]\||=|\|Q\|| .
$$

Proof: We know that $\|Q\|\left\|^{2}=\sum_{k}\right\| \mathbf{q}^{k} \|^{2}$; by inspection we see that $\|B D[Q]\| \|^{2}=$ $\sum_{k} \sum_{i} \sum_{k}\left|q_{i j}^{k}\right|^{2}=\sum_{k}\left\|\mathbf{q}^{k}\right\|^{2}$; hence the result.

THEOREM 5. If $H$ has finite dimension $N$, then the space of all $H S B D$ matrices of size $N^{2} \times N^{2}$ is a normed matrix algebra (with a unit element), isometric-isomorphic to the $\mathrm{H}^{*}$-algebra $(H S Q(H), \bullet)$.

Proof: The result is trivial if $N=1$, so assume $N>1$. The result will then follow once we have set up an identification between the elements $q_{i j}^{k}$ of the 2-matrix representation of $Q$ and the elements $b_{r}^{*}$ in the diagonal blocks of the block diagonal matrix $B D[Q]$. Thus, corresponding to $q_{i j}^{k}$ we have $b_{r}^{*}$ where $r=$ $k(N-1)+i, s=k(N-1)+j$, uniquely. Conversely, given any $b_{r}^{s} \in B D[Q]$, if $\operatorname{Int}(r /(N-1))=\operatorname{Int}(s /(N-1))$ (where $\operatorname{Int}(x)$ is the greatest integer $\leqslant$ the real number $x$ ) then their common value will be $k$ and hence $i=r-k(N-1), j=s-k(N-1)$, which determines $q_{i j}^{k}$; otherwise $b_{r}^{*}$ is outside every diagonal block, has value 0 , and corresponds to no element $q_{i j}^{k}$, in either case the identification is unique.

REMARK. It is the impracticality of determining the bijective relationship between the $r, s$ and $i, j, k$ indices when $N=\infty$ which prevents the result in Theorem 4 from being extended to an infinite dimensional Hilbert space $H$. This is similar to the impracticality which arises when attempting to enumerate a countably infinite matrix as a sequence: it cannot be done 'row-by-row' even though we know it can via 'finite diagonals'.

Corollary. If $H$ has finite dimension $N$, then the $\mathrm{H}^{*}$-algebra $(H S Q(H), \bullet)$ of quadratic Hilbert-Schmidt operators is isometric-isomorphic to a sub-algebra (of dimension $N^{2} \times N$ ) of the $\mathrm{H}^{*}$-algebra $L\left(\bigoplus_{k \in I} H\right.$ ) (of dimension $N^{2} \times N^{2}$ ) of linear operators on the Hilbert sum (of dimension $N^{2}$ ) of $N$ copies of the Hilbert space $H$. The sub-algebra is proper if $N>1$.

Proof: The $H S B D$ matrix representation of $Q$ shows at once that each $Q$ can be identified with a linear operator on the finite-dimensional Hilbert sum $\bigoplus_{k \in I} H$, and hence $H S B D$ can be identified with a (closed) sub-algebra of the $\mathrm{H}^{*}$-algebra of all linear operators on $\bigoplus_{k \in I} H$. A matrix algebra has a basis consisting of all matrices each with precisely one non-zero component; counting these for the algebra and its sub-algebra of block-diagonal matrices gives their stated dimensions. 


\section{REFERENCES}

[1] W. Ambrose, 'Structure theorems for a special class of Banach Algebras', Trans. Amer. Math. Soc 57 (1945), 364-386.

[2] J.C. Amson, 'Multimatrix polynomial representations of the polar composition of polynomial operations', Proc. London Math. Soc. (3) XXV (1972), 465-485.

[3] J.C. Amson, 'Representations of multilinear and polynomial operations on vector spaces', J. London Math Soc. (2) 4 (1972), 394-400.

[4] J. Bochnak and J. Siciak, 'Polynomials and multilinear mappings in topological vector spaces', Studia Math. 39 (1971), 59-76.

[5] N. Dunford and J.T. Schwartz, Linear Operators, Part II : Spectral Theory (Interscience, London, 1963).

[6] I.M. Gel'fand and N. Ya. Vilenkin, Generalized functions, volume \&, Applications of harmonic analysis (Academic Press, London, 1964). (Translated from the Russian edition 1961).

[7] W.H. Greub, Multilinear Algebra (Springer-Verlag, Berlin, Heidelberg, New York, 1967). (2nd Edition 1978).

[8] P.M. Prenter, 'On polynomial operators and equations', in Nonlinear functional analysis and applications, (Mathematics Center, University of Wisconsin, Madison) Editor L.B. Rall (Academic Press, London, 1971).

[9] R. Schatten, Norm ideals of completely continuous operators: Erg. d. Math. 27 (SpringerVerlag, Berlin, Heidelberg, New York (2nd printing), 1970).

[10] J. Weidman, Linear operators in Hilbert space (Springer-Verlag, Berlin, Heidelberg, New York, 1980).

Department of Mathematical Sciences

The Mathematical Institute

University of St Andrews

St Andrews Fife Ky16 9SS

Scotland

United Kingdom
Department of Mathematics

Osmania University

H No 9-29 Ravindar Nagar Colony

Habisguda, Hyderabad 500007

India 